\title{
Monotherapy with Vancomycin or Daptomycin versus Combination Therapy with $\beta$-Lactams in the Treatment of Methicillin-Resistant Staphylococcus Aureus Bloodstream Infections: A Retrospective Cohort Analysis
}

Sara Alosaimy · Noor L. Sabagha · Abdalhamid M. Lagnf · Evan J. Zasowski · Taylor Morrisette •

Sarah C. J. Jorgensen · Trang D. Trinh · Ryan P. Mynatt · Michael J. Rybak

Received: February 13, 2020 / Published online: April 4, 2020

(C) The Author(s) 2020

\section{ABSTRACT}

Background: Methicillin-resistant Staphylococcus aureus (MRSA) bloodstream infections (BSI) are associated with high morbidity and mortality. More in vitro, in vivo, and clinical data suggest that vancomycin (VAN) or daptomycin (DAP) combination therapy with $\beta$-lactams (BL) improves outcomes of MRSA infections. We hypothesize that BL combination with VAN or DAP would reduce the odds of clinical failure compared to VAN or DAP monotherapy.

Methods: A retrospective cohort study of adult patients $\geq 18$ years treated with VAN or DAP for MRSA BSI from 2006 to 2019 at Detroit Medical

Enhanced Digital Features To view digital features for this article go to https://doi.org/10.6084/m9.figshare. 11973288.

S. Alosaimy · A. M. Lagnf - T. Morrisette .

M. J. Rybak ( $\square)$

Eugene Applebaum College of Pharmacy and Health Sciences, Wayne State University, Detroit, MI, USA e-mail:m.rybak@wayne.edu

N. L. Sabagha

Department of Pharmacy, Henry Ford Hospital,

Detroit, MI, USA

E. J. Zasowski

Department of Clinical Sciences, Touro University

California College of Pharmacy, Vallejo, CA, USA

S. C. J. Jorgensen

Mount Sinai Hospital, Toronto, ON, Canada
Center. Combination therapy (CT) was defined as VAN or DAP plus any BL for $\geq 24 \mathrm{~h}$ within $72 \mathrm{~h}$ of index culture. Monotherapy (MT) was defined as $\geq 72 \mathrm{~h}$ VAN or DAP within $72 \mathrm{~h}$ of index culture and no BL for $\geq 24 \mathrm{~h}$ up to 7 days following VAN/DAP initiation. Primary outcome was composite endpoint of clinical failure defined as: (1) 30-day mortality, (2) 60-day recurrence, or (3) persistent bacteremia (PB). PB was defined as bacteremia $>5$ days. Multivariable logistic regression was used to evaluate the association between CT and the primary outcome.

Results: Overall, 597 patients were included in this analysis, 153 in the MT group and 444 in the CT group. CT was independently associated with reduced odds of clinical failure (adjusted odds ratio, $0.523 ; 95 \%$ confidence interval, 0.348-0.787). The composite endpoint was

\section{T. D. Trinh}

Department of Clinical Pharmacy, San Francisco

School of Pharmacy, University of California, San Francisco, CA, USA

\section{R. P. Mynatt}

University of Kentucky Healthcare, Lexington, KY, UK

\section{J. Rybak}

Department of Pharmacy, Detroit Medical Center,

Detroit, MI, USA

\section{J. Rybak}

School of Medicine, Wayne State University,

Detroit, MI, USA 
driven by 60-day recurrence and $\mathrm{PB}$ but not 30-day mortality. There were no difference in adverse events including nephrotoxicity between the two study arms.

Conclusions: In hospitalized adults with MRSA BSI, CT with any BL was independently associated with improved clinical outcomes and may ultimately be selected as preferred therapy.

Keywords: $\beta$-lactams;

Gram-positive infections; Vancomycin

\section{Key Summary Points}

Combination therapy was independently associated with reduced odds of clinical failure in methicillin-resistant Staphylococcus aureus bloodstream infections.

The composite endpoint of clinical failure was driven by 60-day recurrence and persistent bacteremia but not 30-day mortality.

Time to bacterial clearance was shorter in patients managed with combination therapy compared to monotherapy.

\section{BACKGROUND}

Methicillin-resistant Staphylococcus aureus (MRSA) is a major public health concern causing serious community and health-care-associated infections annually [1-3]. Mortality rates associated with MRSA bloodstream infections (BSI) can be as high as 57\% [3]. For decades, vancomycin (VAN) has been the mainstay for the management of MRSA BSI despite complex dosing strategies, nephrotoxicity risk, and slower bactericidal rate [4]. Daptomycin (DAP) is an alternative agent that offers solutions to VAN pitfalls; however; clinical outcomes for DAP treated patients, especially with Food and Drug Administration (FDA)-approved doses, are not superior to VAN plus gentamicin [5]. Furthermore, some $S$. aureus mutations encode DAP

resistance and permit enhanced survival characteristics while on DAP treatment [6]. Interestingly, none of the novel agents for MRSA have been shown to be superior to VAN for MRSA BSI [5, 7].

Combination therapy (CT) with an active $\beta$ lactam (BL) early in the course of MRSA BSI has been suggested as a possible treatment strategy due to observed synergy between glycopeptides and BLs [8-17]. This phenomenon has been termed the "see-saw" effect; where, in the presence of glycopeptide or lipoglycopeptide, the susceptibility to BLs improves [18-21]. Additionally, this strategy achieves higher bactericidal activity, enables use of lower VAN or DAP doses in vitro and may even allow de-escalation to one agent $[13,17,22,23]$. This approach had been utilized for the clinical management of MRSA BSI, particularly if adopted early in therapy $[8-11,16,17,22,24-26]$. While it had been quite promising with regards to faster microbiological eradication, the impact on other clinical outcomes, particularly mortality, had been underwhelming due to the scarcity of quality clinical evidence $[8-11,15,16,25,27]$. On a more sobering note, VAN CT with some BL agents, specifically flucloxacillin and piperacillin-tazobactam, had been associated with an increased risk of acute kidney injury (AKI) $[9,15,28,29]$. We sought to determine whether CT improves clinical outcomes and safety compared to VAN or DAP monotherapy (MT) in patients with MRSA BSI.

\section{METHODS}

\section{Study Design and Population}

This was a retrospective cohort study at the Detroit Medical Center (DMC) between 2006 and 2019. The DMC is a single large healthsystem of eight hospitals including six centered in midtown Detroit. Patients were screened and eligible for inclusion upon meeting the following criteria: (1) age $\geq 18$ years; (2) MRSA-positive blood culture meeting Centers for Disease Control and Prevention criteria for BSI [30], and (3) treated with VAN or DAP within $72 \mathrm{~h}$ of index culture for $\geq 72 \mathrm{~h}$. Patients were classified 
in the MT group if they did not receive any BL for $\geq 24 \mathrm{~h}$ up to 7 days following VAN/DAP initiation. Patients were in the CT group if they received $\mathrm{BL}$ for $\geq 24 \mathrm{~h}$ within $72 \mathrm{~h}$ after VAN/ DAP initiation. Patients were excluded if they (1) experienced polymicrobial BSI, (2) did not have follow-up blood cultures, (3) a second MRSA BSI episode $<90$ days of first episode, (4) cleared their bacteremia prior to MT or CT initiation, or (5) switched to ceftaroline $\geq 72 \mathrm{~h}$ following MT or CT (Fig. 1). The study was reviewed and approved by the Wayne State University Human Investigational Review Board and the DMC Research Review Committee.

\section{Data Collection and Study Definitions}

Demographics, comorbid conditions, laboratory, clinical and treatment data, infectious disease consult, and the pursuit of source control were extracted from the electronic medical record and entered into REDCap (Research Electronic Data Capture, Vanderbilt Univeristy), an electronic data capture tool hosted at Wayne State University [31]. Blood cultures were processed at the DMC microbiology laboratories according to standard procedures. MicroScan (Siemans Healthcare Diagnostics), Phoenix (BD), or Vitek2 (bioMerieux) were used for

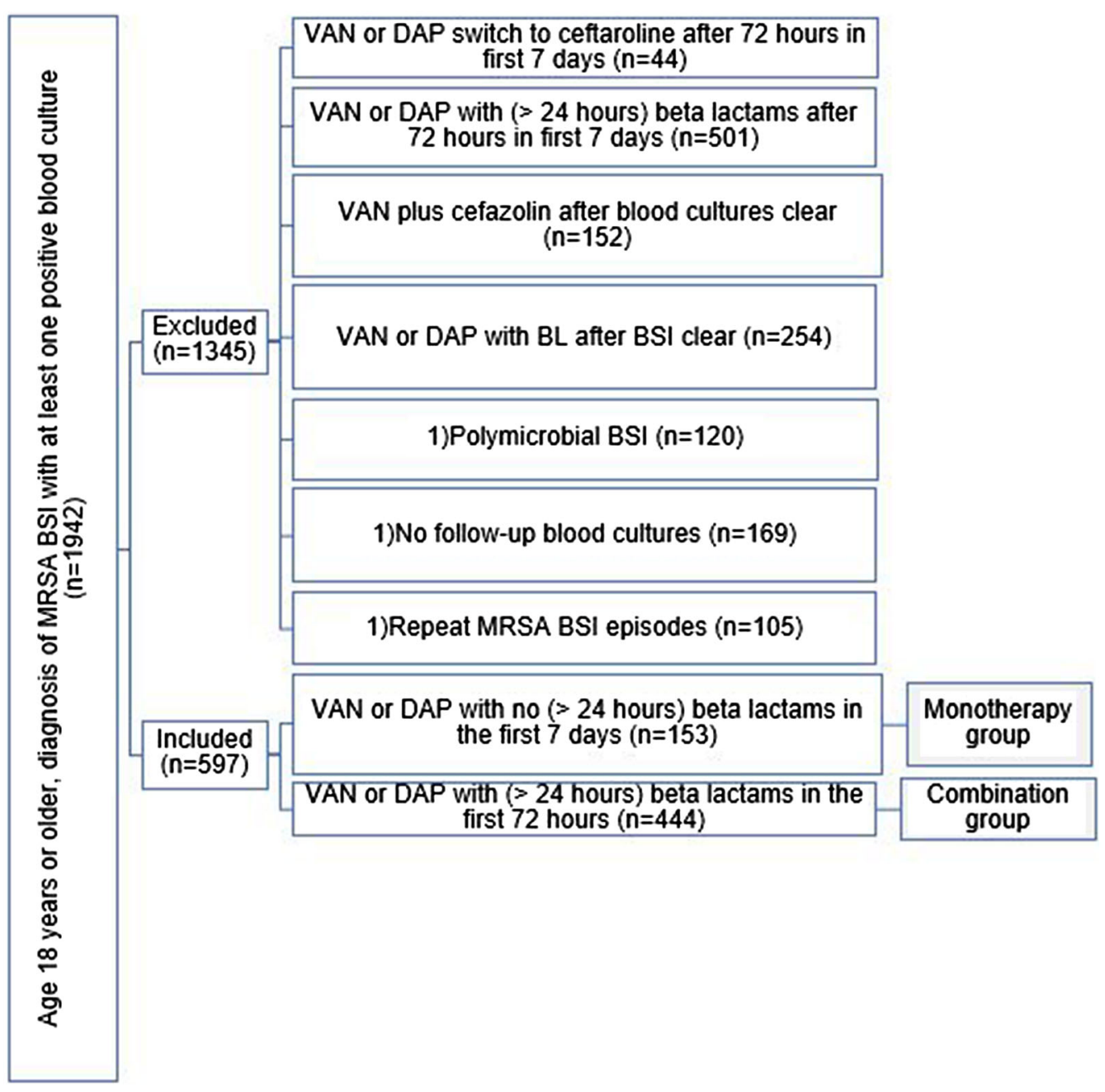

Fig. 1 Patient enrollment and selection. Exclusion criteria: (1) experienced polymicrobial BSI, (2) did not have followup blood cultures, (3) a second MRSA BSI episode. BSI blood stream infections, DAP daptomycin, $V A N$ Vancomycin, MRSA methicillin-resistant Staphylococcal aureus 
bacterial identification and antimicrobial susceptibility testing. The source of BSI was determined based on clinical notes and microbiological/diagnostic reports. Infective endocarditis was defined according to the modified Duke criteria [32]. BSI was classified as hospital-acquired if the index blood culture was obtained $\geq 48 \mathrm{~h}$ after hospital admission. The Charlson Comorbidity Index (CCI) was used to measure the degree of patient comorbidity [33]. The severity of illness was quantified using the Acute Physiology and Chronic Health evaluation II (APACHE II) score at BSI onset [34].

\section{Outcome}

The primary outcome was composite failure defined as (1) 30-day mortality, (2) 60-day recurrence, or (3) persistent BSI. Recurrence was defined as the development of recurrent positive culture after apparent clinical cure within 90 days of discharge. Persistent BSI was defined as BSI $\geq 5$ days [35]. All time points were measured from the index MRSA blood culture. Secondary outcomes of interest included the individual component of the composite outcome, nephrotoxicity, hospital readmission, length of stay and other safety outcomes. Nephrotoxicity was defined as an increase in serum creatinine $(0.5 \mathrm{mg} / \mathrm{dl}$ or $\geq 50 \%$ increase from baseline, whichever was greater). Other safety outcomes included thrombocytopenia, rhabdomyolysis and Clostridioides difficile-associated diarrhea.

\section{Statstical Analysis}

Demographics were evaluated using descriptive statistics where nominal data were reported as percentages and frequencies, and continuous data were reported as medians and interquartile ranges (IQR). Categorical variables between the success and failure group were compared by the Chi squared or Fisher's exact tests, and continuous variables were compared by the Student's $t$ test or Mann-Whitney $U$ test, as appropriate. Multivariable logistic regression was performed to examine the independent association between $\mathrm{CT}$ and clinical failure while adjusting for confounding variables. CT and monotherapy, alone with all baseline variables associated with clinical failure in the bivariate analysis at $P<0.2$ were included in the logistic regression models simultaneously and removed using a backward stepwise approach. Variables that were not deemed to be independent were not included. The variance inflation factor was used to assess the multicollinearity of candidate regression. Values in the range of $1-5$ were deemed to be appropriate. Covariates were retained in the model if the $P$ value for the likelihood ratio test for their removal was $<0.1$. Model fit was assessed with the Hosmer-Lemeshow goodness of fit test. All tests were two tailed with $P$ values $\leq 0.5$ to be considered significant. IBM SPSS software, ve.6.0 (SPSS, Chicago, IL, USA) was used for all calculations.

\section{RESULTS}

\section{Study Population}

Our database identified 1942 patients in whom MRSA BSI and VAN/DAP had been completed for $\geq 72 \mathrm{~h}$. Of these patients, 1345 cases were excluded (Fig. 1). Overall, the median age of this sample was 59 years (IQR, 50, 68), the median Charlson Comorbidity and APACHE II scores were $3(1,5)$ and $17(11,23)$, respectively. The majority were male sex (64.8\%), African American (78.9\%) and were admitted from home $(72.5 \%)$. The most common comorbidities were diabetes (38.9\%), chronic kidney disease (35.0\%), and chronic dialysis (25.3\%). More than one-third were hospitalized for at least $48 \mathrm{~h}$ within the past 90 days (38.2\%). Most common sources of BSI were skin and soft tissue infections $(21.7 \%)$, followed by infective endocarditis (19.4\%) and bone and joint infections (17.9\%).

The majority of patients were on CT therapy $[n=444(74.4 \%)]$ and a quarter of the patients received MT [ $n=153(25.6 \%)]$. A comparison of baseline characteristics between these two groups is provided in Table 1. No MRSA BSI patients with pneumonia as a source were treated with DAP in either cohort arms. The two 
Table 1 Bivariate comparison of clinical characteristics and outcomes of patients managed with monotherapy versus combination therapy

\begin{tabular}{|c|c|c|c|}
\hline Characteristics $^{\mathrm{a}}$ & $\begin{array}{l}\text { Monotherapy (VAN/ } \\
\text { DAP) } \\
(n=153)\end{array}$ & $\begin{array}{l}\text { Combination therapy VAN or DAP }+\beta- \\
\text { lactam } \\
(n=444)\end{array}$ & $P$ value \\
\hline \multicolumn{4}{|l|}{$\overline{\text { Demographics }}$} \\
\hline Age in years, mean $( \pm S D)$ & $56.7(15.7)$ & $59.3(16.1)$ & 0.079 \\
\hline Age over 60 years, $n(\%)$ & $49(32.0)$ & $199(44.8)$ & 0.006 \\
\hline Male sex, $n(\%)$ & $99(64.7)$ & $288(64.9)$ & 0.972 \\
\hline \multicolumn{4}{|l|}{ Race, $n(\%)$} \\
\hline African American & $128(83.7)$ & $343(77.3)$ & 0.094 \\
\hline Caucasian & $21(13.7)$ & $90(20.3)$ & 0.073 \\
\hline Hispanic & $2(1.3)$ & $4(0.9)$ & 0.664 \\
\hline Other/unknown & $2(1.3)$ & $5(0.01)$ & 0.858 \\
\hline \multicolumn{4}{|l|}{ Admission source, $n$ (\%) } \\
\hline Home & $117(76.5)$ & $316(71.2)$ & 0.205 \\
\hline Nursing facility & $25(16.4)$ & $102(23.0)$ & 0.084 \\
\hline Transfer from outside institution & $11(7.2)$ & $26(5.8)$ & 0.555 \\
\hline \multicolumn{4}{|l|}{ Primary BSI source, $n(\%)$} \\
\hline Pneumonia/LRT & $8(5.2)$ & $98(22.1)$ & $<0.001$ \\
\hline Skin/soft tissue & $24(15.7)$ & $55(12.4)$ & 0.299 \\
\hline Intra-abdominal & $0(0)$ & $4(0.9)$ & 0.239 \\
\hline Infective endocarditis & $28(18.3)$ & $98(22.0)$ & 0.682 \\
\hline Intravenous catheter & $28(17.9)$ & $77(17.3)$ & 0.904 \\
\hline Bone and joint & $40(26.1)$ & $62(14.0)$ & 0.001 \\
\hline Prosthetic device & $11(7.2)$ & $22(5)$ & 0.297 \\
\hline Urinary & $3(2)$ & $10(2.3)$ & 0.831 \\
\hline CNS & $6(3.9)$ & $6(1.4)$ & 0.051 \\
\hline Others or unknown & $26(17.0)$ & $81(18.2)$ & 0.728 \\
\hline \multicolumn{4}{|l|}{ Comorbid conditions, $n$ (\%) } \\
\hline Myocardial infarction & $6(3.9 \%)$ & $37(8.3)$ & 0.069 \\
\hline Chronic pulmonary disease ${ }^{\mathrm{b}}$ & $25(16.3)$ & $94(21.2)$ & 0.197 \\
\hline Dementia & $10(6.5)$ & $51(11.49)$ & 0.081 \\
\hline Diabetes & $57(37.3)$ & $175(39.4)$ & 0.637 \\
\hline With end organ damage ${ }^{c}$ & $44(28.8)$ & $124(27.9)$ & 0.844 \\
\hline Peripheral vascular disease & $32(20.5)$ & $79(17.8)$ & 0.497 \\
\hline CVA (stroke or TIA) & $21(13.7)$ & $75(16.9)$ & 0.358 \\
\hline Heart failure & $31(20.3)$ & $94(21.2)$ & 0.811 \\
\hline Moderate to severe $\mathrm{CKD}^{\mathrm{d}}$ & $47(30.7)$ & $162(36.5)$ & 0.197 \\
\hline Chronic dialysis & $38(24.8)$ & $113(25.5)$ & 0.880 \\
\hline HIV & $7(4.6)$ & $13(2.9)$ & 0.348 \\
\hline AIDS & $3(1.9)$ & $3(0.6)$ & 0.169 \\
\hline Any immunosuppression factor & $11(7.2)$ & $22(5)$ & 0.297 \\
\hline Liver disease & $30(19.6)$ & $52(11.7)$ & 0.014 \\
\hline Moderate/severe $\mathrm{e}^{\mathrm{e}}$ & $2(1.3)$ & $9(2.0)$ & 0.568 \\
\hline
\end{tabular}


Table 1 continued

\begin{tabular}{|c|c|c|c|}
\hline Characteristics $^{\mathrm{a}}$ & $\begin{array}{l}\text { Monotherapy (VAN/ } \\
\text { DAP })(n=153)\end{array}$ & $\begin{array}{l}\text { Combination therapy VAN or DAP }+\beta \text { - } \\
\operatorname{lactam}(n=444)\end{array}$ & $P$ value \\
\hline \multicolumn{4}{|l|}{ MRSA BSI risk factors, $n(\%)$} \\
\hline Person with injection drug use & $31(20.3)$ & $68(15.3)$ & 0.195 \\
\hline Prior hospitalization $>48$ in preceding 90 days & $54(35.3)$ & $174(39.2)$ & 0.392 \\
\hline Prior surgery 30 days preceding index culture & $16(10.3)$ & $29(6.5)$ & 0.113 \\
\hline $\begin{array}{l}\text { Prior MRSA infection } \leq 365 \text { days preceding index } \\
\text { culture }\end{array}$ & $34(22.2)$ & $54(12.2)$ & 0.002 \\
\hline Prior antibiotics $\geq 24 \mathrm{~h}$ in preceding 90 days & $60(39.2)$ & $150(33.8)$ & 0.232 \\
\hline \multicolumn{4}{|l|}{ Severity of illness factors } \\
\hline APACHE II score, mean $[ \pm S D)$ & $13.7(7.2)$ & $19.1(9.2)$ & $<0.001$ \\
\hline ICU at index culture, $\mathrm{n}(\%)$ & $15(9.8)$ & $78(17.6)$ & 0.022 \\
\hline CCI score, mean $[ \pm S D)$ & $3.1(2.4)$ & $3.1(2.4)$ & 0.561 \\
\hline \multicolumn{4}{|l|}{$\beta$-lactam } \\
\hline Cefepime & $\mathrm{N} / \mathrm{A}$ & $204(45.9)$ & NA \\
\hline Cefazolin & $\mathrm{N} / \mathrm{A}$ & $149(33.6)$ & NA \\
\hline Ceftaroline & NA & $54(12.2)$ & NA \\
\hline Piperacillin/tazobactam & NA & $68(15.3)$ & NA \\
\hline Ceftriaxone & NA & $85(15.3)$ & NA \\
\hline Ampicillin/sulbactam & NA & $14(3.2)$ & NA \\
\hline Meropenem & NA & $28(6.3)$ & NA \\
\hline Others ${ }^{\mathrm{f}}$ & NA & $7(1.6)$ & NA \\
\hline \multicolumn{4}{|l|}{ Glycopeptide/lipopeptide } \\
\hline VAN only & $83(54.2)$ & $258(58.1)$ & 0.405 \\
\hline DAP only & $10(6.5)$ & $11(2.5)$ & 0.019 \\
\hline VAN and DAP & $60(39.2)$ & $175(39.4)$ & 0.965 \\
\hline Duration of VAN, days, median (IQR) & $6.1(4.4-11.0)$ & $6.0(4.3-9.2)$ & $<0.001$ \\
\hline Duration of DAP, days, median (IQR) & $10.2(7.3-15.3)$ & $8.5(6.0-14.4)$ & 0.021 \\
\hline Removable source of infection & $81(52.9)$ & $180(40.5)$ & 0.009 \\
\hline ID consult & $130(85.0)$ & $391(88.1)$ & 0.322 \\
\hline \multicolumn{4}{|l|}{ Daptomycin dose, mg } \\
\hline $6-8 \mathrm{mg}$ per day & $17(24.3)$ & $62(33.3)$ & 0.162 \\
\hline $8-10 \mathrm{mg}$ per day & $29(41.4)$ & $68(36.6)$ & 0.493 \\
\hline$>10 \mathrm{mg}$ per day & $17(24.3)$ & $38(20.4)$ & 0.516 \\
\hline \multicolumn{4}{|l|}{ Clinical outcomes } \\
\hline Clinical failure & $80(52.3)$ & $195(43.9)$ & 0.073 \\
\hline 30-day mortality & $14(9.2)$ & $67(15.1)$ & 0.064 \\
\hline 60-day recurrence & $22(14.4)$ & $39(8.8)$ & 0.049 \\
\hline Persistent bacteremia $^{\mathrm{h}}$ & $59(40.0)$ & $118(27.5)$ & 0.005 \\
\hline BSI duration, days, median (IQR) & $4.2(2.5-7.5)$ & $3.3(2.0-5.3)$ & 0.003 \\
\hline Length of stay, days, median (IQR) & $12(8-19)$ & $10(7-16)$ & 0.087 \\
\hline 60-day readmission & $62(40.5)$ & $149(33.6)$ & 0.134 \\
\hline Safety & & & \\
\hline
\end{tabular}


Table 1 continued

\begin{tabular}{llll}
\hline Characteristics $^{\mathbf{a}}$ & $\begin{array}{l}\text { Monotherapy (VAN/ } \\
\text { DAP })(\boldsymbol{n}=\mathbf{1 5 3})\end{array}$ & $\begin{array}{l}\text { Combination therapy VAN or DAP + } \boldsymbol{\beta} \text { - } \\
\text { lactam }(\boldsymbol{n}=\mathbf{4 4 4})\end{array}$ \\
\hline Nephrotoxicity $^{\mathrm{I}}$ & $22(14.4)$ & $63(14.2)$ & 0.954 \\
Thrombocytopenia & $21(13.7)$ & $75(16.9)$ & 0.358 \\
Clostridium difficile Diarrhea $^{\mathrm{j}}$ & $4(2.6)$ & $18(4.0)$ & 0.415 \\
\hline
\end{tabular}

AIDS acquired immune deficiency virus, $A P A C H E$ II acute physiology and chronic health evaluation II, $B S I$ blood stream infection, $C C I$ charlson comorbidity score, $C N S$ central nervous system, $H I V$ human immunodeficiency syndrome, $I D$ infectious diseases, $I C U$ intensive care unit, $B S I$ bloodstream infection, $L R T$ lower respiratory tract infection, $M I C$ minimum inhibitory concentration, $M R S A$ methicillinresistant Staphylococcus aureus

a All values represent number (\%) or median (interquartile range)

b Asthma and chronic obstructive pulmonary disease

c End-organ damage includes diabetic nephropathy, neuropathy, and retinopathy

d Chronic kidney disease stages III-IV

e Moderate/severe liver disease defined as portal hypertension or cirrhosis

f Other $\beta$-lactams may include other carbapenems, monobactams and cephalosporins

g Patients may have been treated with vancomycin followed by daptomycin during the same treatment course

h Denominator have changed as patients experiencing mortality before blood stream infection clearance were amended; combination therapy $(n=429)$ and monotherapy $(n=148)$

I Nephrotoxicity defined as serum creatinine increase of $0.5 \mathrm{mg} / \mathrm{L}$ and $50 \%$ from baseline on two consecutive measurements from initial antibiotic exposure to $72 \mathrm{~h}$ after the last dose

j Clostridioides difficile infection defined as signs/symptoms of infection with positive laboratory test at least $48 \mathrm{~h}$ after the initiation of study antibiotics

groups were similar in general with a few notable differences. The CT group had a greater proportion of patients $>60$ years old, admitted from a nursing home, with pneumonia, endocarditis or primary bacteremia as a source, and had higher APACHE II scores. The MT group had a greater proportion of patients with a MRSA infection history, recent antibiotic usage, and bone/joint or skin and soft tissue as a bacteremia source. Various BL agents were utilized in the CT group with the most common being cefepime $204(45.9 \%)$, followed by cefazolin 149 (33.6\%) and ceftaroline 54 (12.2\%). Most patients received an infectious diseases (ID) service consult; $88.1 \%$ and $85.0 \%$ in the MT and CT groups, respectively. Source control was more commonly pursued in the MT $(52.9 \%)$ group compared to CT (40.5\%). Median (IQR) BSI duration was significantly longer in the MT compared to CT group; 4.2 and 3.3 days, respectively $(P<0.003)$. Median (IQR) length of stay was also longer but not statistically significant in the MT group compared to CT; 12 (8, $19)$ and $10(7,16)$ days, respectively $(P=0.087)$.

A total of $275(46.1 \%)$ study patients experienced composite clinical failure; specifically $80(52.3 \%)$ in the MT group compared to the CT
195 (43.9\%) group (unadjusted $P=0.073$ ). Bivariate analysis was conducted between cases that achieved clinical success and clinical failure, the results of the analysis are provided in Table 2. Two components of the composite endpoint were lower in the CT group: 60-day recurrence $8.7 \%$ versus $14.4 \%(P=0.049)$ and PB $28.3 \%$ versus $41.8 \%(P=0.002)$. On the other hand, 30-day mortality favored MT 9.2\% versus $15.0 \%$, however; that was not statistically significant $(P=0.064)$ (Fig. 2$)$.

Results of the multivariable logistic regression analysis for independent predictors of the composite endpoint are illustrated in Table 3. Upon adjusting for the following variables: age $>60$ years, APACHE II scores, admission from home, chronic kidney disease, MRSA risks (surgery within past 30 days and MRSA within 365 days), sources (endocarditis, skin and soft tissue infection, prosthetic device source). CT was independently associated with lower odds of clinical failure [adjusted odds ratio (aOR), $0.545 ; 95 \%$ confidence interval (CI), $0.364-0.817]$. Because PB was the primary driver for the clinical outcome, we also performed a logistic regression analysis with $\mathrm{PB}$ as the dependent variable and adjusted for sources 
Table 2 Clinical characteristics of patients with clinical failure and no clinical failure

\begin{tabular}{llll}
\hline Characteristics $^{a}$ & $\begin{array}{l}\text { Clinical failure } \\
(n=275)\end{array}$ & $\begin{array}{l}\text { No clinical failure } \\
(n=322)\end{array}$ & $P$ value \\
\hline
\end{tabular}

Demographics and comorbid conditions present on admission

Mean age, years $( \pm S D)$

Age over 60, years

No. of male participants (\%)

Admission from home

Admission from nursing

Transfer from outside institution

Primary BSI source

Pneumonia/other lower respiratory tract

Skin/soft tissue

Infective endocarditis

Central nervous system

Bone and joint

Prosthetic device

Urinary

Other or unknown

Comorbidity conditions, no. of patients (\%)

Myocardial infarction

Heart failure

Chronic pulmonary disease ${ }^{\mathrm{b}}$

Dementia

Diabetes

Peripheral vascular disease

CVA (stroke or TIA)

Moderate to severe $\mathrm{CKD}^{\mathrm{c}}$

Chronic dialysis

HIV

Any immunosuppression

Liver disease

MRSA bacteremia risk factors, no. of patients (\%)

Intravenous drug use

Prior hospitalization $>48$ in preceding 90 days
$19(6.9)$

$62(22.5)$

$52(18.9)$

$57(20.7)$

$59.9(15.8)$

$126(45.8)$

$178(64.7)$

189 (68.7)

$66(24.0)$

$20(7.3)$

$55(20.0)$

$19(6.9)$

79 (28.7)

$8(2.9)$

$52(18.9)$

$21(7.6)$

$5(1.8)$

45 (16.4)

31 (11.3)

$112(40.7)$

56 (20.4)

40 (14.5)

109 (39.6)

72 (26.2)

$5(1.8)$

$13(4.7)$

$41(14.9)$

$108(39.3)$
$57.6(16.2)$

0.079

$122(37.9)$

0.050

209 (64.9)

0.963

244 (75.7)

0.054

61 (18.9)

0.132

17 (5.2)

0.314

$51(15.8)$

0.185

60 (18.6)

$<0.001$

37 (11.5)

$<0.001$

$4(1.2)$

0.148

50 (15.5)

0.274

$12(3.7)$

0.037

8 (2.5)

0.578

$62(19.3)$

0.359

$24(7.5)$

0.798

63 (19.6)

0.372

67 (20.8)

0.563

$30(9.3)$

0.432

$120(37.3)$

0.388

54 (16.8)

0.259

56 (17.4)

0.345

$100(31.1)$

0.028

79 (24.5)

0.664

$15(4.6)$

0.055

$20(6.2)$

0.429

41 (12.7)

0.441

42 (13.0)

0.012

$120(37.3)$ 
Table 2 continued

\begin{tabular}{lccr}
\hline Characteristics $^{\mathbf{a}}$ & $\begin{array}{l}\text { Clinical failure } \\
(\boldsymbol{n}=\mathbf{2 7 5})\end{array}$ & $\begin{array}{c}\text { No clinical failure } \\
(\boldsymbol{n}=\mathbf{3 2 2})\end{array}$ & $\boldsymbol{P}$ value \\
\hline Prior surgery 30 days preceding index culture & $16(5.8)$ & $29(9.0)$ & 0.141 \\
Prior MRSA infection $\leq 365$ days preceding index culture & $48(17.5)$ & $40(12.4)$ & 0.084 \\
Prior antibiotics $\geq 24$ in preceding 90 days & $94(34.2)$ & $116(36.0)$ & 0.618 \\
Severity of illness markers & & & \\
APACHE II score, $( \pm$ SD) & $19.29(9.6)$ & $16.32(8.3)$ & 0.001 \\
ICU at index culture, $n(\%)$ & $49(17.8)$ & $44(13.7)$ & 0.163 \\
Charlson Comorbidity score, $( \pm$ SD) & $3.26(2.4)$ & $2.92(2.3)$ & 0.087 \\
Daptomycin dose, mg & $37(30.1)$ & $42(31.1)$ & 0.927 \\
6-8 mg per day & $42(34.7)$ & $55(40.7)$ & 0.321 \\
8-10 mg per day & $27(22.3)$ & $28(20.7)$ & 0.760 \\
$>10$ mg per day & & & 0.893 \\
Source control, no. of patients $(\%)$ & $121(44.0)$ & $140(43.5)$ & 0.807 \\
Removable source of infection & $239(86.9)$ & $281(87.6)$ & \\
ID consult &
\end{tabular}

$A I D S$ acquired immune deficiency virus, $A P A C H E I I$ acute physiology and chronic health evaluation II, $B S I$ blood stream infection, $C C I$ charlson comorbidity score, $C N S$ central nervous system, $I D$ infectious diseases, $I C U$ intensive care unit, $B S I$ bloodstream infection, $M I C$ minimum inhibitory concentration, MRSA methicillin-resistant Staphylococcus aureus, HIV acquired immunodeficiency syndrome

a All values represent number (\%) or median (interquartile range) as indicated

b Asthma and chronic obstructive pulmonary disease

c Chronic kidney disease stages III-IV

(endocarditis, pneumonia, bone and joint, invasive prosthetic device), source control, and comorbid condition of connective tissue disease. CT was independently associated with reduced odds of $\mathrm{PB}(\mathrm{aOR}, 0.597 ; 95 \% \mathrm{CI}$, 0.393-0.907). We provide the results of the model in Table 4.

We did not observe a difference in nephrotoxicity between the CT and MT groups 14.2\% and $14.4 \%$, respectively $(P=0.954)$. Among subjects who were on VAN only, nephrotoxicity was comparable between CT and MT patients; $10.1 \%$ and $15.6 \%$, respectively $(P=0.164)$. No differences in other secondary safety outcomes such as thrombocytopenia, rhabdomyolysis and
Clostridioides difficile-associated diarrhea were observed between the two groups.

\section{DISCUSSION}

In response to emerging evidence regarding the synergy between BL and VAN/DAP in MRSA BSI treatment, we aimed to explore the association between BL combination with VAN or DAP [8-11, 13-15, 19, 22, 24-26]. Our findings support what has been demonstrated in vitro and in vivo, suggesting that the addition of BL to VAN/DAP therapy within $72 \mathrm{~h}$ for at least $24 \mathrm{~h}$ increases the odds of clinical success. Favorable clinical outcomes occurred in the CT group even upon adjustment for all confounding 


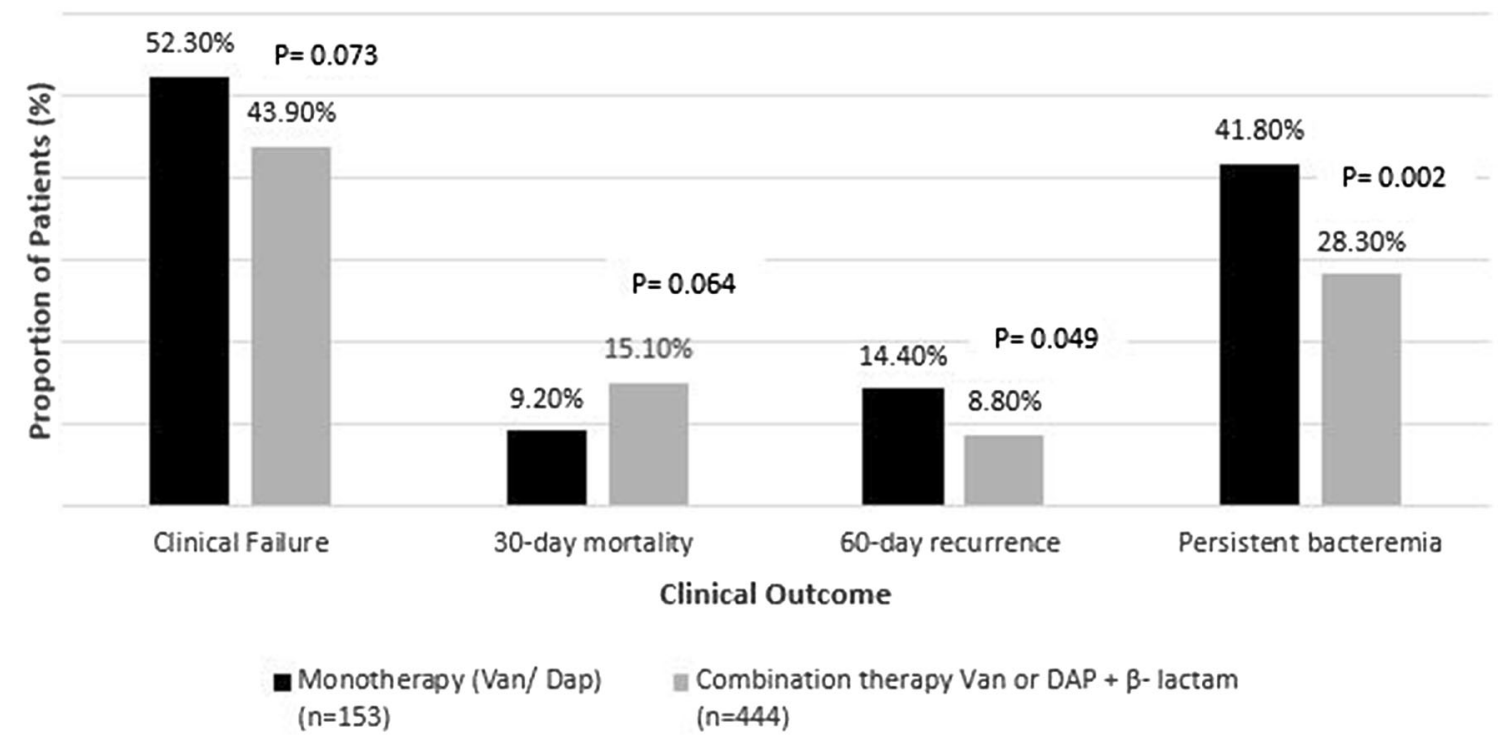

Fig. 2 Comparison of clinical outcomes between monotherapy and combination therapy patients. DAP daptomycin, Van vancomycin

variables in the multivariable regression model. Interestingly, the clinical success was primarily driven by significant reductions in 60-day recurrence and shorter BSI duration ( $<5$ days).

BL addition to VAN/DAP has been shown to have a significant impact on BSI duration compared with MT, with some influence on the rate of composite clinical outcomes such as mortality, relapse, and/or change in antibiotic therapy during treatment [8, 16, 26, 36-38]. Specifically, median duration of bacteremia had been reduced from 4 to 3 days when BL were used with VAN, and from 2.8 to 2.3 days when BL were used with DAP $[8,26]$. Notably, our definition for $\mathrm{PB}$ at a 5-day cut-off has prevented us from comparing our results to other studies that have used other definitions such as 3 days and 7 days. In other observational studies, ceftaroline when used as salvage therapy in addition to DAP decreased the duration of bacteremia from 10 to 2 days [36]. In another study, reduction in BSI duration was more profound when ceftaroline was used with DAP than DAP MT 4.8 days versus 9.3 days, respectively [27]. However, statistically significant mortality differences were not observed between the two treatment groups. Notably, CT patients had a higher APACHE II score, intensive care unit (ICU) encounter, were more likely to be admitted from nursing facilities and had BSI sources commonly associated with high mortality (i.e., pneumonia/LRT and infective endocarditis). This further complicates the conflicting evidence in regard to survival benefits with BL combination therapy particularly with rapid bacterial eradication $[8,9,16,25,37,38]$.

In our study, CT subjects had higher severity of illness and more complex infections than MT subjects, and were therefore at a high risk for mortality. As demonstrated in previous studies, high-risk MRSA BSI patients benefit the most from CT when administered early in the treatment course (i.e., within $72 \mathrm{~h}$ ) $[8,36,39]$. This underscores the importance of early BL addition to achieve timely bacterial eradication (i.e., 3-4 days) and hence avoiding the "perfect storm" [29]. The actual impact of timely bacterial eradication on mortality is well studied but not yet definitive [38].

The majority of BLs were administered as part of empiric treatment and not for targeted synergy against MRSA. Nevertheless, our CT group represents a heterogeneous group of $\mathrm{BL}$ agents administered within the initial $72 \mathrm{~h}$ of VAN/DAP initiation. Nephrotoxicity and other safety outcomes were similar between both groups, and remarkably comparable to previous 
Table 3 Multivariable logistic regression model for factors independently associated with clinical failure

\begin{tabular}{lll}
\hline Variable & $\begin{array}{l}\text { Unadjusted odd ratio } \\
(\mathbf{9 5 \%} \mathbf{C I})\end{array}$ & $\begin{array}{c}\text { Adjusted odds ratio } \\
(\mathbf{9 5 \%} \text { CI })\end{array}$ \\
\hline Source & $3.369(2.127-5.336)$ & $3.294(2.115-5.132)$ \\
Endocarditis & $0.775(0.499-1.203)$ & \\
Skin and soft tissue & $1.746(0.796-3.829)$ & \\
Prosthetic device & $1.434(0.884-2.326)$ & \\
Pneumonia & $1.190(0.807-1.754)$ & $1.545(0.954-2.500)$ \\
Co-morbidity & $1.566(0.956-2.563)$ & \\
CKD & $0.716(0.365-1.406)$ & \\
Prior MRSA in 365 days & & \\
Prior surgery in 365 days & $0.775(0.521-1.154)$ & \\
Other factors & $1.222(0.844-1.769)$ & \\
Admission, home & $1.034(1.011-1.058)$ & $0.545(0.364-0.817)$ \\
Age $>60$ years & & $(1.029-1.072)$ \\
APACHE II score & $0.539(0.356-0.816)$ & \\
Treatment &
\end{tabular}

Hosmer-Lemeshow goodness of fit test $P=0.983$; variance inflation factor $=1.0-1.5$ for all variables included at model entry

$A P A C H E I I$ acute physiology and chronic health evaluation II, CKD chronic kidney disease

studies [16, 40]. Notably, because our study includes VAN- and DAP-treated patients, it may reduce our ability to detect VAN-associated nephrotoxicity. Because our institutions avoid piperacillin-tazobactam/VAN combinations and utilize cefepime/VAN instead, due to the wellknown association with nephrotoxicity, only a small proportion of $\mathrm{CT}$ patients in our study have received piperacillin-tazobactam, and most did in combination with DAP [28]. This may have contributed to the lower nephrotoxicity rates observed in our cohort, which is crucial, particularly in the light of the CAMERA2 study where higher nephrotoxicity had been observed when VAN was used in combination with flucloxacillin.

To our knowledge, this is the largest realworld study to date comparing clinical outcomes in MRSA BSI patients receiving VAN/DAP
MT to VAN/DAP CT with BL. Strengths of this study include a specific definition of BL exposure with regards to VAN/DAP timing, exclusion of patients who have not had a follow-up blood culture, robust sample size captured in over 12 years duration, and findings that are confirmatory of previously published data in CT for MRSA BSI $[8,25,26]$.

When interpreting these findings, there are several considerations to note. First, although this was a multi-hospital site study, it was restricted to hospitals within a single healthcare system in the Detroit area. Therefore, it may be challenging to generalize these findings to other patient populations with different demographics and clinical practice patterns. These hospitals, as of 2016, have mandated an infectious disease consult for all MRSA-positive blood cultures, which may have had an impact 
Table 4 Multivariable logistic regression for factors independently associated with persistent bacteremia

\begin{tabular}{lll}
\hline Variable & $\begin{array}{l}\text { Unadjusted odd ratio } \\
(\mathbf{9 5 \%} \mathbf{C I})\end{array}$ & $\begin{array}{l}\text { Adjusted odds ratio } \\
\mathbf{( 9 5 \% ~ C I ) ~}\end{array}$ \\
\hline Source & $3.342(2.128-5.247)$ & $3.331(2.132-5.205)$ \\
Endocarditis & $0.639(0.396-1.032)$ & $0.629(0.391-1.014)$ \\
Skin and soft tissue & $0.597(0.317-1.124)$ & \\
Pneumonia & $1.488(0.921-2.406)$ & $1.517(0.943-2.440)$ \\
Bone and joint & $1.366(0.614-3.040)$ & \\
Prosthetic device & $1.236(0.828-1.844)$ & \\
Other factors & & \\
Source control & & \\
Treatment & $0.604(0.397-0.918)$ & $0.597(0.393-0.907)$ \\
Combination therapy
\end{tabular}

Hosmer-Lemeshow goodness of fit test $P=0.980$; variance inflation factor $=1.0-1.27$ for all variables included at model entry

on the clinical outcomes. Second, because this was a retrospective study, results may be challenged by inherent limitations associated with this study design and unmeasured confounders. Recognizing this limitation, objective and measurable outcomes were selected (i.e., blood culture clearance and 30-day mortality). Furthermore, the MT arm was predominately treated with VAN or VAN followed by DAP and not DAP alone: $54.2 \%, 39.2 \%$, and $6.5 \%$, respectively. Despite that these two agents are similar in many aspects including spectrum of activity, these two antibiotics are not equivalent and, therefore, our results may be more generalizable to VAN or VAN/DAP rather than DAP alone. Additionally, it was not possible to account for VAN exposure in the entire cohort since the area under the curve (AUC) monitoring strategy was initiated within the last 4 years of the study period [41]. However, it is unlikely that this small proportion of patients have impacted the composite endpoint, as VAN exposure using AUC monitoring is a known factor to influence VAN safety outcomes rather than clinical outcomes [42]. Moreover, although ceftaroline was the BL of choice for only a small proportion of CT patients (12\%); ceftaroline is the only $\mathrm{BL}$ available in the United States to date with in vitro MRSA activity providing known synergy particularly with DAP [22]. This may have had an indirect impact on $\mathrm{PB}$ rates within the CT group. However, it remains unclear if MT with ceftaroline alone would be superior to MT with VAN/DAP or even CT with BL. Finally, because our study evaluates a diverse group of BLs with diverse therapeutic characteristics, it is challenging to draw specific conclusions with regard to which specific BL is preferred when considering CT for MRSA BSI treatment.

\section{CONCLUSION}

We found that $\mathrm{BL}$ given $\leq 72 \mathrm{~h}$ of VAN/DAP therapy improves composite clinical outcomes for patients with MRSA BSI and that CT was well tolerated compared to MT alone. Time to bacterial clearance was shorter in patients managed with CT compared to MT. These data add to the evidence suggesting that CT may improve overall patient outcomes and should be further investigated to ascertain its role in MRSA BSI treatment. Additional studies, particularly prospective analysis, are warranted to explore the relationship between BL selection in CT and clinical benefit. 


\section{ACKNOWLEDGEMENTS}

Funding. No funding or sponsorship was received for this study or publication of this article.

Authorship. All named authors meet the International Committee of Medical Journal Editors (ICMJE) criteria for authorship for this article, take responsibility for the integrity of the work as a whole, and have given their approval for this version to be published.

Prior Presentation. This study has been presented, in part, at American Society for Microbiology (ASM); October 3 -7, 2018 San Francisco, CA (abstract 2379) and the following publications Zasowski et al. and Jorgensen et al. $[25,26]$.

Disclosures. Michael J. Rybak: Research support, consultant or speaker for Allergan, Melinta, Merck, Motif, Nabriva, Paratek, Tetraphase and Shionogi. Michael J. Rybak is the Editor-in-Chief of this journal. Sara Alosaimy, Noor L. Sabagha, Abdalhamid M. Lagnf, Evan J. Zasowski, Taylor Morrisette, Sarah C.J. Jorgensen, Trang D. Trinh and Ryan P. Mynatt have nothing to disclose.

Compliance with Ethics Guidelines. The study was reviewed and approved by the Wayne State University Human Investigational Review Board and the DMC Research Review Committee.

Data Availability. The datasets during and/ or analyzed during the current study are available from the corresponding author on reasonable request.

Open Access. This article is licensed under a Creative Commons Attribution-NonCommercial 4.0 International License, which permits any non-commercial use, sharing, adaptation, distribution and reproduction in any medium or format, as long as you give appropriate credit to the original author(s) and the source, provide a link to the Creative Commons licence, and indicate if changes were made. The images or other third party material in this article are included in the article's Creative Commons licence, unless indicated otherwise in a credit line to the material. If material is not included in the article's Creative Commons licence and your intended use is not permitted by statutory regulation or exceeds the permitted use, you will need to obtain permission directly from the copyright holder. To view a copy of this licence, visit http://creativecommons.org/licenses/by$\mathrm{nc} / 4.0 /$.

\section{REFERENCES}

1. Boucher HW, Talbot GH, Bradley JS, Edwards JE, Gilbert D, Rice LB, et al. Bad bugs, no drugs: no ESKAPE! An update from the Infectious Diseases Society of America. Clin Infect Dis. 2009;48(1): $1-12$.

2. Lodise TP, McKinnon PS. Clinical and economic impact of methicillin resistance in patients with Staphylococcus aureus bacteremia. Diagn Microbiol Infect Dis. 2005;52(2):113-22.

3. Lamagni TL, Potz N, Powell D, Pebody R, Wilson J, Duckworth G. Mortality in patients with meticillinresistant Staphylococcus aureus bacteraemia, England 2004-2005. J Hosp Infect. 2011;77(1):16-20.

4. Deresinski S. Counterpoint: vancomycin and Staphylococcus aureus-an antibiotic enters obsolescence. Clin Infect Dis. 2007;44(12):1543-8.

5. Fowler VG Jr, Boucher HW, Corey GR, Abrutyn E, Karchmer AW, Rupp ME, et al. Daptomycin versus standard therapy for bacteremia and endocarditis caused by Staphylococcus aureus. N Engl J Med. 2006;355(7):653-65.

6. Berti AD, Shukla N, Rottier AD, McCrone JS, Turner HM, Monk IR, et al. Daptomycin selects for genetic and phenotypic adaptations leading to antibiotic tolerance in MRSA. J Antimicrob Chemother. 2018;73(8):2030-3.

7. Wilcox MH, Tack KJ, Bouza E, Herr DL, Ruf BR, Ijzerman MM, et al. Complicated skin and skinstructure infections and catheter-related bloodstream infections: noninferiority of linezolid in a phase 3 study. Clin Infect Dis. 2009;48(2):203-12.

8. Casapao AM, Jacobs DM, Bowers DR, Beyda ND, Dilworth TJ. Early administration of adjuvant $\beta$ lactam therapy in combination with vancomycin 
among patients with methicillin-resistant Staphylococcus aureus bloodstream infection: a retrospective, multicenter analysis. Pharmacotherapy. 2017;37(11):1347-56.

9. Davis JS, Sud A, O'Sullivan MVN, Robinson JO, Ferguson PE, Foo $\mathrm{H}$, et al. Combination of vancomycin and $\beta$-lactam therapy for methicillin-resistant Staphylococcus aureus bacteremia: a pilot multicenter randomized controlled trial. Clin Infect Dis. 2016;62(2):173-80.

10. Dilworth TJ, Sliwinski J, Ryan K, Dodd M, Mercier RC. Evaluation of vancomycin in combination with piperacillin-tazobactam or oxacillin against clinical methicillin-resistant Staphylococcus aureus Isolates and vancomycin-intermediate $S$. aureus isolates in vitro. Antimicrob Agents Chemother. 2014;58(2):1028-33.

11. Geriak M, Haddad F, Rizvi K, Rose W, Kullar R, LaPlante $\mathrm{K}$, et al. Clinical data on daptomycin plus ceftaroline versus standard of care monotherapy in the treatment of methicillin-resistant Staphylococcus aureus bacteremia. Antimicrob Agents Chemother. 2019;63(5):e02483-18.

12. Sieradzki K, Tomasz A. Suppression of $\beta$-lactam antibiotic resistance in a methicillin-resistant Staphylococcus aureus through synergic action of early cell wall inhibitors and some other antibiotics. J Antimicrob Chemother. 1997;39(Suppl A):47-51.

13. Tran KN, Rybak MJ. $\beta$-lactam combinations with vancomycin show synergistic activity against vancomycin-susceptible Staphylococcus aureus, vancomycin-intermediate $S$. aureus (VISA), and heterogeneous VISA. Antimicrob Agents Chemother. 2018;62(6):e00157-18.

14. Hagihara M, Wiskirchen DE, Kuti JL, Nicolau DP. In vitro pharmacodynamics of vancomycin and cefazolin alone and in combination against methicillin-resistant Staphylococcus aureus. Antimicrob Agents Chemother. 2012;56(1):202-7.

15. Tong SYC, Lye DC, Yahav D, Sud A, Robinson JO, Nelson J, et al. Effect of vancomycin or daptomycin with vs without an antistaphylococcal $\beta$-lactam on mortality, bacteremia, relapse, or treatment failure in patients with MRSA bacteremia: a randomized clinical trial. JAMA. 2020;323(6):527-37.

16. Truong J, Veillette JJ, Forland SC. Outcomes of vancomycin plus a $\beta$-lactam versus vancomycin only for treatment of methicillin-resistant Staphylococcus aureus bacteremia. Antimicrob Agents Chemother. 2018;62(2):e01554-17.

17. McConeghy KW, Bleasdale SC, Rodvold KA. The empirical combination of vancomycin and a $\beta$ - lactam for Staphylococcal bacteremia. Clin Infect Dis. 2013;57(12):1760-5.

18. Ortwine JK, Werth BJ, Sakoulas G, Rybak MJ. Reduced glycopeptide and lipopeptide susceptibility in Staphylococcus aureus and the "seesaw effect": taking advantage of the back door left open? Drug Resist Updat. 2013;16(3-5):73-9.

19. Barber KE, Ireland CE, Bukavyn N, Rybak MJ. Observation of "seesaw effect" with vancomycin, teicoplanin, daptomycin and ceftaroline in 150 unique MRSA strains. Infect Dis Ther. 2014;3(1): $35-43$.

20. Renzoni A, Kelley WL, Rosato RR, Martinez MP, Roch M, Fatouraei M, et al. Molecular bases determining daptomycin resistance-mediated resensitization to $\beta$-lactams (seesaw effect) in methicillinresistant Staphylococcus aureus. Antimicrob Agents Chemother. 2017;61(1):e01634-16.

21. Jousselin A, Manzano C, Biette A, Reed P, Pinho $\mathrm{MG}$, Rosato AE, et al. The Staphylococcus aureus chaperone PrsA is a new auxiliary factor of oxacillin resistance affecting penicillin-binding protein $2 \mathrm{~A}$. Antimicrob Agents Chemother. 2015;60(3): 1656-66.

22. Barber KE, Werth BJ, Rybak MJ. The combination of ceftaroline plus daptomycin allows for therapeutic de-escalation and daptomycin sparing against MRSA. J Antimicrob Chemother. 2015;70(2):505-9.

23. Sakoulas G, Okumura CY, Thienphrapa W, Olson J, Nonejuie P, Dam Q, et al. Nafcillin enhances innate immune-mediated killing of methicillin-resistant Staphylococcus aureus. J Mol Med (Berlin, Germany). 2014;92(2):139-49.

24. Werth BJ, Sakoulas G, Rose WE, Pogliano J, Tewhey $\mathrm{R}$, Rybak MJ. Ceftaroline increases membrane binding and enhances the activity of daptomycin against daptomycin-nonsusceptible vancomycinintermediate Staphylococcus aureus in a pharmacokinetic/pharmacodynamic model. Antimicrob Agents Chemother. 2013;57(1):66-73.

25. Zasowski EJ, Trinh TD, Atwan SM, Merzlyakova M, Langf AM, Bhatia S, et al. The impact of concomitant empiric cefepime on patient outcomes of methicillin-resistant Staphylococcus aureus bloodstream infections treated with vancomycin. Open Forum Infect Dis. 2019;6(7):ofz077.

26. Jorgensen SCJ, Zasowski EJ, Trinh TD, Lagnf AM, Bhatia S, Sabagha N, et al. Daptomycin plus $\beta$-lactam combination therapy for methicillin-resistant Staphylococcus aureus bloodstream infections: a retrospective, comparative cohort study. Clin Infect Dis. 2019. https://doi.org/10.1093/cid/ciz746. 
27. McCreary EK, Kullar R, Geriak M, Zasowski EJ, Rizvi $\mathrm{K}$, Schulz LT, et al. Multicenter cohort of patients with methicillin-resistant Staphylococcus aureus bacteremia receiving daptomycin plus ceftaroline compared with other MRSA treatments. Open Forum Infect Dis 2019. https://doi.org/10.1093/ ofid/ofz538.

28. Navalkele B, Pogue JM, Karino S, Nishan B, Salim M, Solanki S, et al. Risk of acute kidney injury in patients on concomitant vancomycin and piperacillin-tazobactam compared to those on vancomycin and cefepime. Clin Infect Dis. 2017;64(2): 116-23.

29. Kullar R, McKinnell JA, Sakoulas G. Avoiding the perfect storm: the biologic and clinical case for reevaluating the 7-day expectation for methicillinresistant Staphylococcus aureus bacteremia before switching therapy. Clin Infect Dis. 2014;59(10): 1455-61.

30. Horan TC, Andrus M, Dudeck MA. CDC/NHSN surveillance definition of health care-associated infection and criteria for specific types of infections in the acute care setting. Am J Infect Control. 2008;36(5):309-32.

31. Harris PA, Taylor R, Thielke R, Payne J, Gonzalez N, Conde JG. Research electronic data capture (REDCap)-a metadata-driven methodology and workflow process for providing translational research informatics support. J Biomed Inform. 2009;42(2): 377-81.

32. Durack DT, Lukes AS, Bright DK. New criteria for diagnosis of infective endocarditis: utilization of specific echocardiographic findings. Duke Endocarditis Service. Am J Med. 1994;96(3):200-9.

33. Charlson ME, Pompei P, Ales KL, MacKenzie CR. A new method of classifying prognostic comorbidity in longitudinal studies: development and validation. J Chronic Dis. 1987;40(5):373-83.

34. Knaus WA, Draper EA, Wagner DP, Zimmerman JE. APACHE II: a severity of disease classification system. Crit Care Med. 1985;13(10):818-29.
35. Neuner EA, Casabar E, Reichley R, McKinnon PS. Clinical, microbiologic, and genetic determinants of persistent methicillin-resistant Staphylococcus aureus bacteremia. Diagn Microbiol Infect Dis. 2010;67(3):228-33.

36. Sakoulas G, Moise PA, Casapao AM, Nonejuie P, Olson J, Okumura CY, et al. Antimicrobial salvage therapy for persistent staphylococcal bacteremia using daptomycin plus ceftaroline. Clin Ther. 2014;36(10):1317-33.

37. Zasowski EJ, Trinh TD, Claeys KC, Casapao AM, Sabagha N, Lagnf AM, et al. Multicenter observational study of ceftaroline fosamil for methicillinresistant Staphylococcus aureus bloodstream infections. Antimicrob Agents Chemother. 2017;61(2): e02015-6.

38. Minejima E, Mai N, Bui N, Mert M, Mack WJ, She $\mathrm{RC}$, et al. Defining the breakpoint duration of Staphylococcus aureus bacteremia predictive of poor outcomes. Clin Infect Dis. 2020;70(4):566-73.

39. Cortes-Penfield N, Oliver NT, Hunter A, RodriguezBarradas M. Daptomycin and combination daptomycin-ceftaroline as salvage therapy for persistent methicillin-resistant Staphylococcus aureus bacteremia. Infect Dis (Lond). 2018;50(8):643-7.

40. Dilworth TJ, Ibrahim O, Hall P, Sliwinski J, Walraven $C$, Mercier RC. $\beta$-Lactams enhance vancomycin activity against methicillin-resistant Staphylococcus aureus bacteremia compared to vancomycin alone. Antimicrob Agents Chemother. 2014;58(1):102-9.

41. Heil EL, Claeys KC, Mynatt RP, Hopkins TL, Brade $\mathrm{K}$, Watt I, et al. Making the change to area under the curve-based vancomycin dosing. Am J Health Syst Pharm. 2018;75(24):1986-95.

42. Ghosh N, Chavada R, Maley M, van Hal SJ. Impact of source of infection and vancomycin AUC0-24/ MICBMD targets on treatment failure in patients with methicillin-resistant Staphylococcus aureus bacteraemia. Clin Microbiol Infect. 2014;20(12): O1098-105. 